\title{
Using Rheo-Small-Angle Neutron Scattering to Understand How Functionalised Dipeptides Form Gels
}

\author{
Kate McAulay ${ }^{a}$ \\ Lisa Thomson ${ }^{a}$ \\ Lionel Porcar ${ }^{b}$ \\ Ralf Schweins $\mathbf{s}^{\mathbf{b}}$ (i) \\ Najet Mahmoudic (i) \\ Dave J. Adams*a (1) \\ Emily R. Draper*a (1) \\ a School of Chemistry, University of Glasgow, Glasgow, G12 8QQ, United Kingdom \\ b Large Scale Structures Group, Institut Laue-Langevin, 71 Avenue des Martyrs, CS \\ 20156, F-38042 Grenoble, CEDEX 9, France \\ 'ISIS Pulsed Neutron Source, Rutherford Appleton Laboratory, Didcot, OX11 0QX, \\ United Kingdom \\ dave.adams@glasgow.ac.uk; Emily.draper@glasgow.ac.uk
}

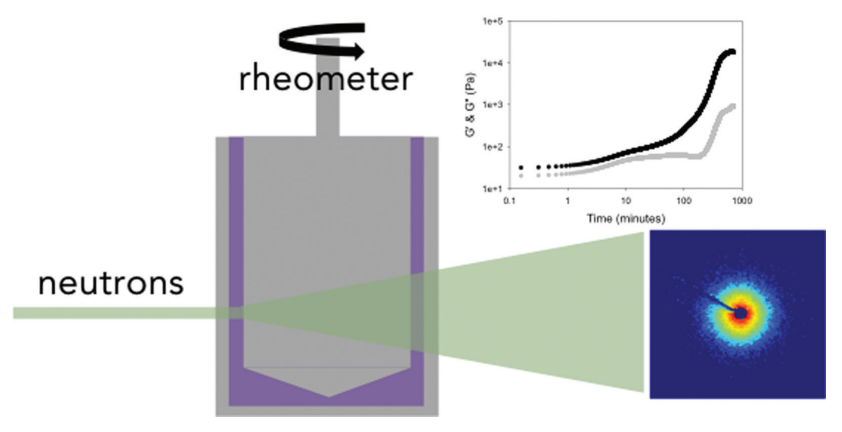

example, many of the functionalised dipeptides can be dissolved in a water-miscible solvent such as DMSO and water added, which leads to the formation of a gel. ${ }^{6,11,17}$

Another common approach is to use a pH trigger. ${ }^{8,18,19}$ Here, the dipeptide is suspended in water and the $\mathrm{pH}$ increased. This deprotonates the terminal carboxylic acid, resulting in a dispersion of the dipeptide. At this point, surfactant-like aggregates are formed, which can be spherical structures (as for $2 \mathrm{NapVG}^{20}$ or $\mathrm{BrNapAV}^{21}$ ) or long anisotropic worm-like micelles or tubes (such as for $2 \mathrm{NapFF}^{20}$ and related diphenylalanine-based materials ${ }^{22}$ ) depending on the structure of the dipeptide. The differences in the aggregates formed in solution can be seen in different ways. For example, the anisotropic structures formed by 2NapFF scatter strongly when probed by small-angle neutron scattering (SANS), whilst the less persistent structures formed by 2NapVG do not. ${ }^{20}$ The viscosities of the solutions are also very different; anisotropic samples often show shear thinning behaviour for example. ${ }^{20}$

Following the suspension of the chosen gelator in water at high $\mathrm{pH}$, the $\mathrm{pH}$ is then decreased to below the apparent $\mathrm{p} K_{\mathrm{a}}$ of the carboxylic acid. ${ }^{23,24}$ This leads to the formation of a selfsupporting gel. ${ }^{21}$ An interesting point is that gels can be formed by dipeptides that form either type of micellar aggregate at high $\mathrm{pH}$. We have explained this as follows. The more hydrophobic dipeptides that form worm-like structures become protonated as the $\mathrm{pH}$ is lowered, leading to a slight morphological change, but maintaining their anisotropic structure and a tendency to aggregate. As the $\mathrm{pH}$ is decreased below the $\mathrm{p} K_{\mathrm{a}}$, entanglement occurs as well as the formation of a (semi-)permanent network (Figure $1 \mathrm{~b}$ ). We have recently shown how this occurs in detail using contrast-matching experiments to understand the assembled structures at high $\mathrm{pH}$ and the transition as the $\mathrm{pH}$ is decreased. ${ }^{25}$ For dipeptides forming non-persistent spherical structures at 
<smiles>CC(C)C(NC(=O)COc1ccc2ccccc2c1)C(=O)NCC(=O)O</smiles>

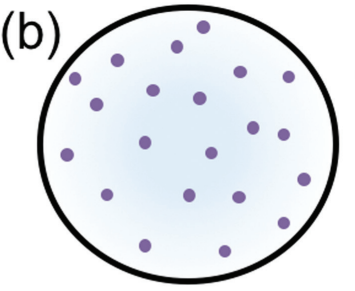

high $\mathrm{pH}$ solution
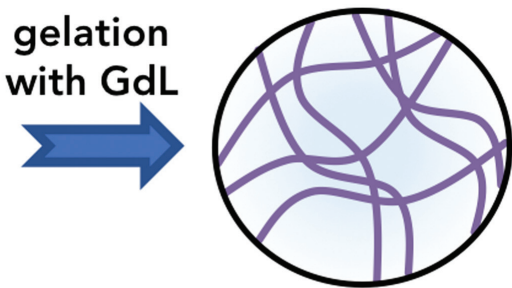

low $\mathrm{pH}$ gel

(c)

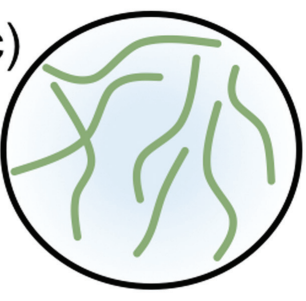

gelation with GdL
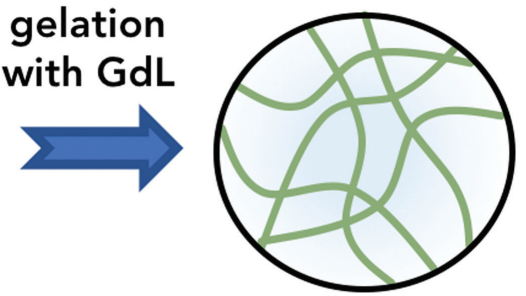

low $\mathrm{pH}$ gel

(d)

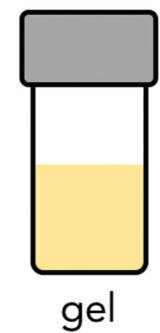

syneresis over time

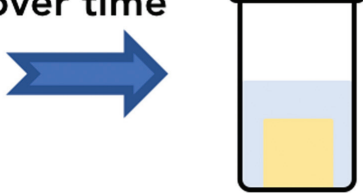

shrunken gel

Figure 1 (a) Chemical structures of 2NapFF, 2NapVG, and BrNapAV. (b) Cartoon showing network formation in the case of 2NapFF. (c) Cartoon showing network formation in the case of 2NapVG. (d) Cartoon showing network formation in the case of BrNapAV.

high $\mathrm{pH}$, there is a transition to worm-like micelles at the $\mathrm{p} K_{\mathrm{a}}$, followed by entanglement and network formation (Figure 1c). ${ }^{26}$

The data obtained so far show how the fibres that lead to the network are formed. An interesting question that remains unanswered for both of these cases is exactly how the network is formed from these fibres and how the network links to the primary structures formed at different $\mathrm{pH}$ values and time. Information as to the selfassembled structures that are formed in these systems can be accessed by SANS. SANS data have been reported for a number of functionalised dipeptides, ${ }^{13,20,27,28}$ showing the presence of different structures at high $\mathrm{pH}$ and in the gel phase. The evolution of the primary self-assembled structures has been followed by SANS. ${ }^{26,28}$ However, it is difficult to access information as to the network from the SANS data alone.

Here, we show rheo-SANS data for both systems, enabling us to directly link the structures formed (probed by SANS) to the mechanical properties of the sample (probed by rheology). Rheo-SANS has been used to monitor a number of gelling systems, as well as solutions containing worm-like micelles. ${ }^{29-31}$ Limited rheo-SANS data have been reported for low-molecular-weight gels previously, although it has recently been noted that a technique such as rheo-SANS would potentially revolutionise the characterisation of supramolecular gels. ${ }^{32}$ Yan et al. have shown rheo-SANS data for hydrogels formed from MAX-1 and MAX-8. ${ }^{33}$ However, no data have been provided to the best of our knowledge for the gelation process itself.

\section{Results and Discussion}

To form our pH-triggered gels, we prepare solutions at high $\mathrm{pH}$ of 2NapVG, 2NapFF, or BrNapAV. We then add glucono- $\delta$-lactone $(\mathrm{GdL})$, which slowly hydrolyses with time to gluconic acid. ${ }^{19,34}$ This results in a slow and uniform $\mathrm{pH}$ change, which allows reproducible gels to be formed. Another advantage of this slow pH change is that this allows the gelation process to be followed with time. ${ }^{19}$ Hence, we are able to follow the gelation process with both rheology and SANS going from the solution state to the gel state.

Focussing initially on the SANS data for 2NapVG, at high $\mathrm{pH}$, the solution scatters weakly. ${ }^{20}$ The data at this 
point can be fitted to a power law. Hence the data indicate that there are large, non-persistent species present. After gelation in a cuvette, the sample scatters much more strongly and the data can be fitted to a flexible elliptical cylinder model. ${ }^{20}$

The gelation process can be followed using rheo-SANS. Here, both rheological and SANS data can be collected simultaneously (Figures 2, S1 and S2). By necessity, an aluminium or titanium concentric cylinder geometry is

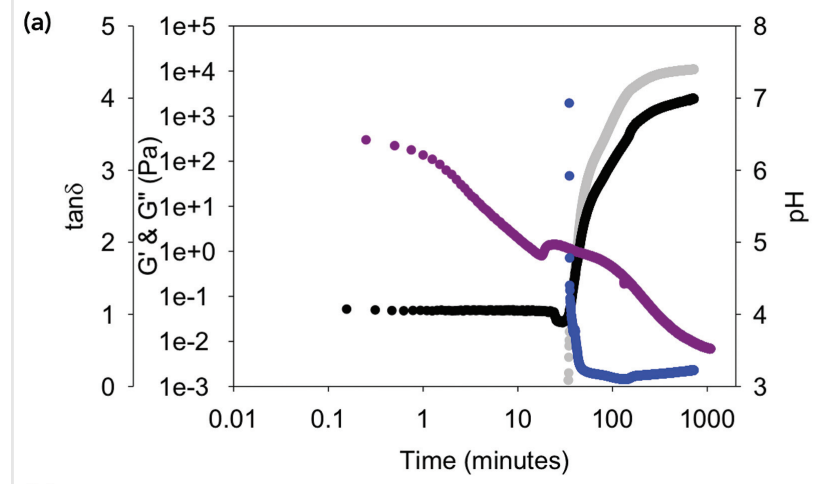

(b)

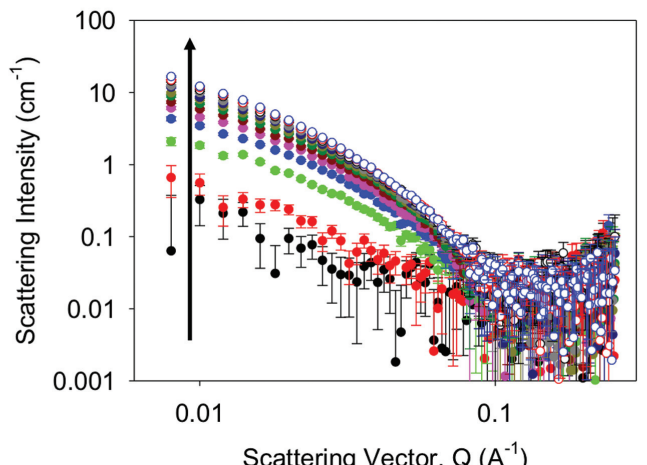

(c)

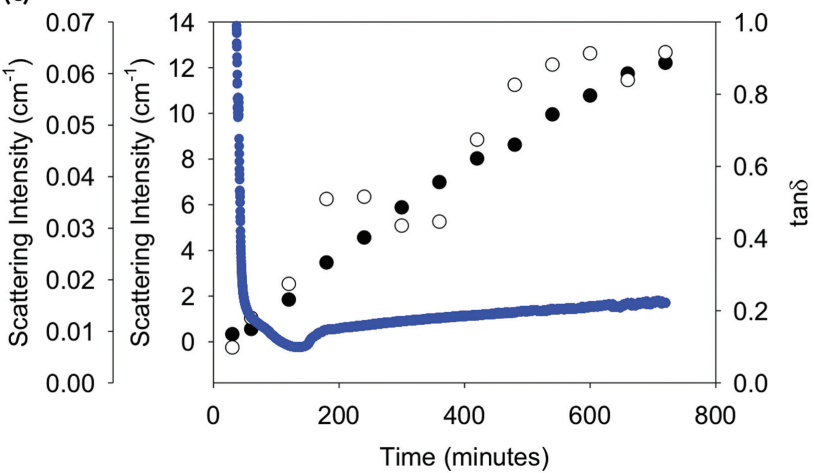

Figure 2 (a) Graph showing the progress of the gelation of 2NapVG over time. Black and grey data represent $G^{\prime}$ and $G^{\prime \prime}$ respectively collected at $0.5 \%$ strain and $10 \mathrm{rad} / \mathrm{s}$. Blue data represent $\tan \delta$ and purple data represent $\mathrm{pH}$. All data were collected at $29^{\circ} \mathrm{C}$. (b) In situ scattering data collected during the gelation in (a), where the arrow shows the trend in the scattering data with time. (c) Plot of scattering intensity at $Q=0.01 \AA^{-1}$ (filled circles) and $Q=0.08 \AA^{-1}$ (open circles) against time as compared to $\tan \delta$ (blue data). used, which allows the neutrons to pass through the sample whilst the gelation is monitored by rheology. The absolute storage modulus $\left(G^{\prime}\right)$ and loss modulus $\left(G^{\prime \prime}\right)$ will be affected by the geometry used, but the trends in the data are the same when the gelation is followed using a parallel plate or a vane geometry.

Initially, as expected from the SANS on the sample in a cuvette, the scattering is very weak (Figures $2 \mathrm{~b}$ and $\mathrm{S} 2 \mathrm{a}, \mathrm{b}$ ). At this point, the rheological data (Figure $2 a$ ) show that $G^{\prime}$ is essentially the same as $G^{\prime \prime}$, indicating that the sample behaves as a liquid. As time progresses, the $\mathrm{pH}$ drops due to the hydrolysis of $\mathrm{GdL}$ and, at pH 5, both $G^{\prime}$ and $G^{\prime \prime}$ begin to increase (Figures 2a and S1a). $G^{\prime}$ and $G^{\prime \prime}$ are very similar in value, but after 30 minutes, $G^{\prime}$ starts to dominate. There is an inflection point in both $G^{\prime}$ and $G^{\prime \prime}$ at 120 minutes, before both begin to reach a plateau at 500 minutes. The change in state can be examined by following $\tan \delta\left(G^{\prime \prime} / G^{\prime}\right)$ with time, with $\tan \delta$ initially being large, indicating a liquid.

After 30 minutes, $\tan \delta$ decreases dramatically such that at 50 minutes (where $G^{\prime}$ becomes significantly larger than $G$ ") it reaches 0.1 (Figures $2 \mathrm{a}$ and $\mathrm{S} 1 \mathrm{~b}$ ). At 2 hours, there is a slight increase in $\tan \delta$ before it begins to plateau after 8 hours, which means a gel network has formed and then is becoming stiffer as the $\mathrm{pH}$ decreases to 3.5 and the assembly progresses (Figure S1c).

Following the SANS over the same time period, initially the scattering is very low. As $G$ ' and $G^{\prime \prime}$ begin to increase, the scattering increases slightly. At 30 minutes and 1 hour, the data can be fitted to a flexible cylinder model (Table S1-S3 and Figure S2c-h). After 2 hours, the data are best fitted to a flexible elliptical cylinder model (Figure S3; note that other models including the flexible cylinder gave poorer fits to the data even when polydispersity was included); at this point, $G$ ' dominates over $G^{\prime \prime}$. As time progresses further, the shape of the scattering data does not change significantly, with there being an increase in scattering intensity until 8 hours; after this time, there are little changes in the data. Overall, from the fits to these data, the absolute length and radius of the scattering objects do not change (although the lengths are around $100 \mathrm{~nm}$ ), and so the model will be relatively insensitive to changes at this length-scale on the basis of the data available. The data were collected over a $Q$ range of $0.007 \leq Q \leq 1.5 \AA^{-1}$, where $Q=(4 p / l) \sin (q / 2)$ with $l$ the neutron wavelength and $q$ the scattering angle. Hence, the data are collected over a length-scale of around 143 to around $1.5 \AA$. Lengths longer than $143 \AA$ can only be inferred from the fits and where there is little change in the data at low $Q$ (longer lengths), the fit becomes relatively insensitive to changes in the length. However, changes in the absolute scattering intensity at low $Q$ can be used to indicate changes in length. As time progresses, the Kuhn length increases gradually indicating that the structures become more rigid over time. The axis ratio also gradually increases with time. 


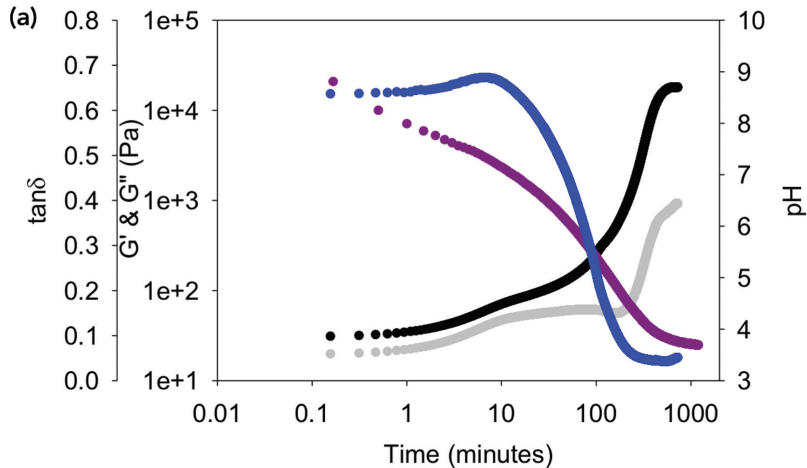

(b)
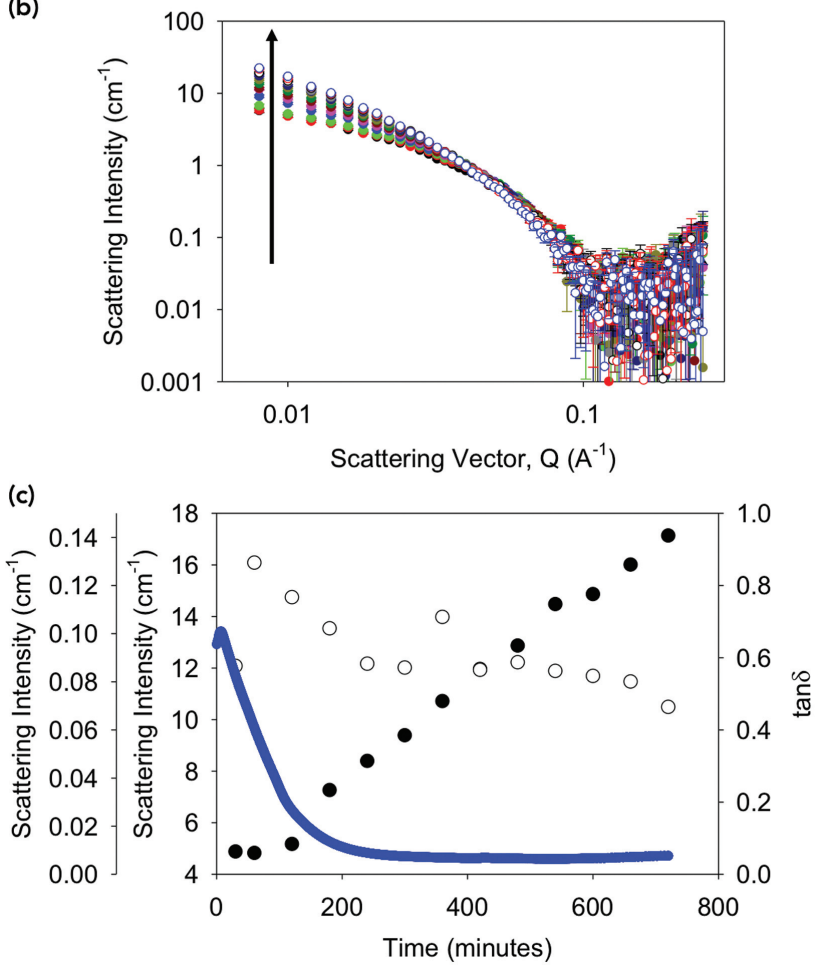

Figure 3 (a) Graph showing the progress of the gelation of 2NapFF over time. Black and grey data represent $G^{\prime}$ and $G^{\prime \prime}$ respectively collected at $0.5 \%$ strain and $10 \mathrm{rad} / \mathrm{s}$. Blue data represent $\tan \delta$ and purple data represent $\mathrm{pH}$. All data were collected at $29^{\circ} \mathrm{C}$. (b) In situ scattering data collected during the gelation in (a), where the arrow shows the trend in the scattering data with time. (c) Plot of scattering intensity at $Q=0.01 \AA^{-1}$ (closed circles) and $Q=0.08 \AA^{-1}$ (open circles) against time as compared to $\tan \delta$ (blue data).

Plotting intensity at $Q=0.01 \AA^{-1}$ against time shows that the scattering increases almost linearly with time (Figure 2c). However, plotting intensity at $Q=0.08 \AA^{-1}$ against time shows a two-stage process, correlating well with the increase in rheological properties (Figure 2c). The intensity at $Q=0.01 \AA^{-1}$ can be related to the increase in length of the scattering objects, whilst that at $Q=0.08 \AA^{-1}$ can be linked to changes in radius and ellipticity. Hence, our interpretation of all of these SANS data is that at early times

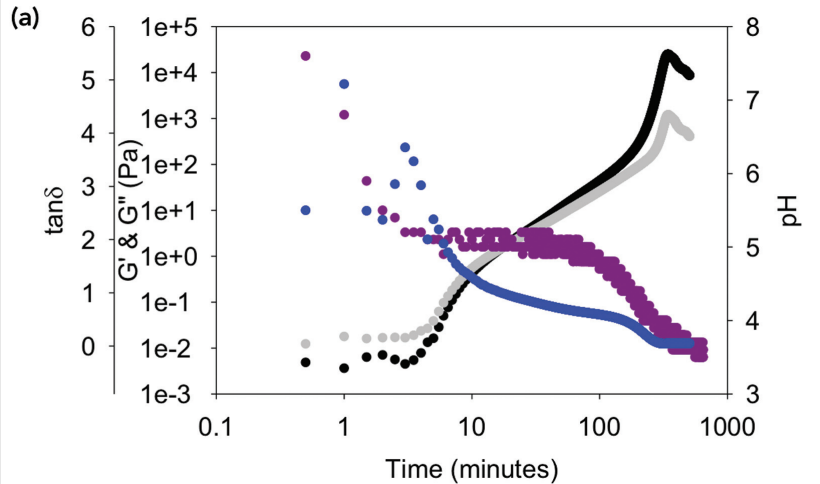

(b)
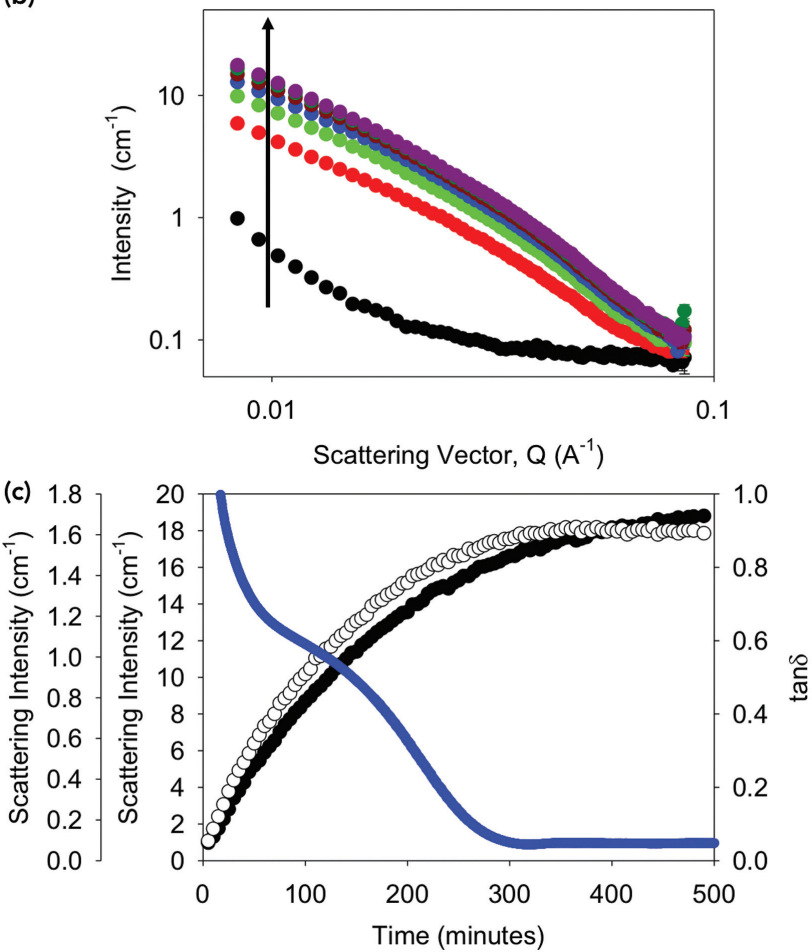

Figure 4 (a) Graph showing the progress of the gelation of BrNapAV over time. Black and grey data represent $G^{\prime}$ and $G^{\prime \prime}$ respectively collected at $0.5 \%$ strain and $10 \mathrm{rad} / \mathrm{s}$. Blue data represent $\tan \delta$ and purple data represent $\mathrm{pH}$. All data were collected at $25^{\circ} \mathrm{C}$. (b) In situ scattering data collected during the gelation in (a), where the arrow shows the trend in the scattering data with time. (c) Plot of scattering intensity at $Q=0.01 \AA^{-1}$ (closed circles) and $Q=0.08 \AA^{-1}$ (open circles) against time as compared to $\tan \delta$ (blue data).

flexible cylinders are formed. These laterally associate with time, which is best captured by an increase in the ellipticity and leads to the structures becoming more rigid.

Moving to the gelator $2 \mathrm{NapFF}$, at high $\mathrm{pH}$ the solution scatters much more strongly than that of $2 \mathrm{NapVG}$. This is due to the presence of persistent, hollow cylinders at high $\mathrm{pH}^{16}$ Again, we followed the gelation process using rheo-SANS (Figures 3a and S4). For this case, $G^{\prime}$ is initially higher than $G^{\prime \prime}$, due to the presence of structures at high $\mathrm{pH}$ leading to viscous 
solutions. As the $\mathrm{pH}$ decreases, with time, $G^{\prime}$ starts to dominate significantly over $G^{\prime \prime}$, with a small increase after 10 minutes ( $\mathrm{pH}$ around 7.2) and a sharp inflection point at 2 hours (around pH 5.4). After 9 hours, $G^{\prime}$ essentially plateaus at $\mathrm{pH}$ 3.8. Again, the change in state can be examined by following $\tan \delta\left(G^{\prime \prime} / G^{\prime}\right)$ with time. $\tan \delta$ shows initially a stable value of 0.7 for 10 minutes, describing a viscous liquid, before a sharp decrease to around 0.06 after 2 hours where it remains at around this value. This illustrates that after 2 hours, the formation of the network has occurred and the gel is just becoming stiffer after this point.

Following the SANS over this time period (Figure $3 \mathrm{~b}$ ), all data can be fitted to an elliptical cylinder (Figures S5 and 6 and Tables S4-6). We have previously shown that there is a rapid change from a hollow cylinder to a cylinder, followed by an increase in ellipticity ${ }^{25}$; this change happens within the first 30 minutes and so is not captured by the current dataset. Over time, the radius remains essentially constant, the length increases, becoming longer than is accessible from the current $Q$ range after 2 hours, with the main change being an increase in ellipticity. After 8 hours, there is little change in the data. Plotting intensity at $Q=0.01$ and $0.08 \AA^{-1}$ against time (Figure $3 \mathrm{c}$ ) shows a different trend to that described above for 2NapVG. There is an increase in the scattering with time $Q=0.01 \AA^{-1}$. However, at $Q=0.08$ $\AA^{-1}$, the scattering intensity remains at around the same value over this time period. Hence, the main changes are the increase in the network over time, with anisotropic structures persisting throughout.

The third example we describe is BrNapAV. This gelator behaves similarly to $2 \mathrm{NapVG}$, starting as a low scattering, nonviscous solution, and gradually forming a gel over time (Figures 4 and S7-S9). ${ }^{21}$ Where BrNapAV differs is the behaviour once gelation has occurred. Here, instead of a stable gel at longer times, there is a tendency for syneresis to occur, whereby the gel network shrinks exuding water, leading to a denser gel in the presence of water (Figures 4a and S7a). This behaviour is clear from the rheology data, where $G^{\prime}$ and $G^{\prime \prime}$ are initially very low, followed by a concomitant increase in both $G^{\prime}$ and $G^{\prime \prime}$ after 4 minutes. There is then a plateau, before $G^{\prime}$ starts to dominate over $G^{\prime \prime}$, which correlates with the $\mathrm{pH}$ buffering around the point of the apparent $\mathrm{p} K_{\mathrm{a}}$ of BrNapAV, before decreasing to 3.6 after 8 hours. After 340 minutes, the syneresis can be observed where both $G^{\prime}$ and $G^{\prime \prime}$ decrease again. This syneresis can be linked to the removal of charge from the self-assembled structures leading to the gel network, and hence the tendency for hydrophobic interactions to lead to the network contracting. ${ }^{17}$ This is confirmed by again looking at $\tan \delta$; this value has stopped increasing after 250 minutes, meaning the gel network has formed, and this value remains unchanged despite the gel syneresis and the $\mathrm{pH}$ continuing to decrease.

Following the SANS over the gelation, initially, there is again very little scattering as expected from the solution
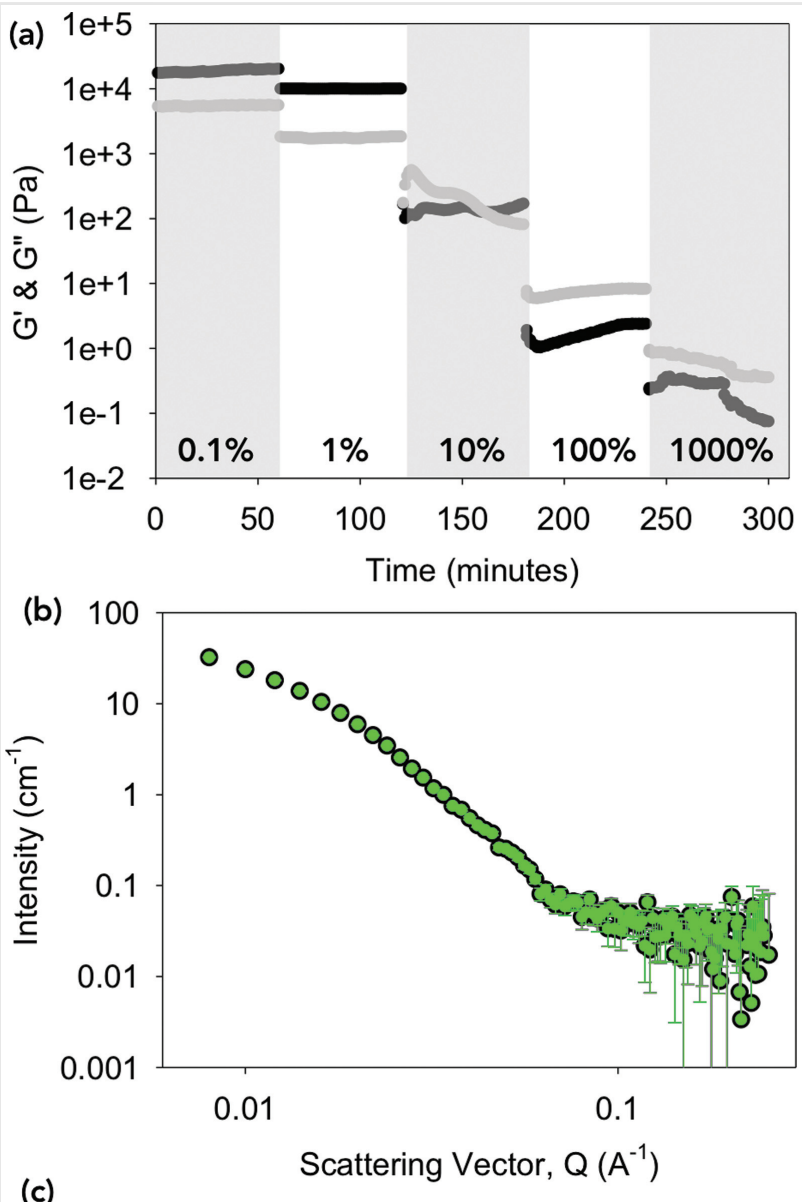

(c)
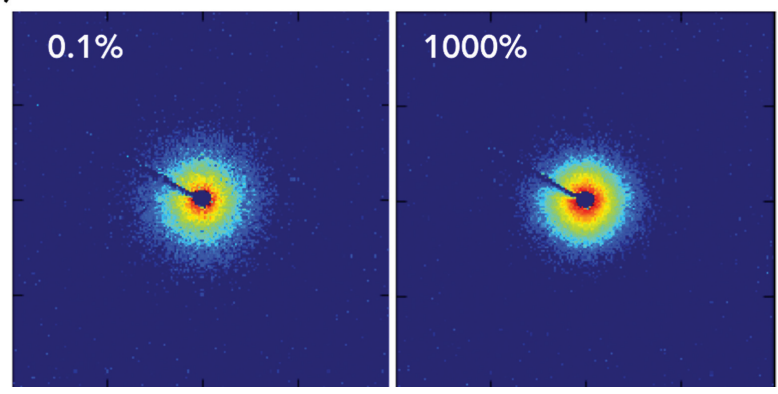

Figure 5 (a) Strain sweep of 2NapVG. Black and grey data represent $G^{\prime}$ and $G^{\prime \prime}$ respectively, collected at $10 \mathrm{rad} / \mathrm{s}$ at $29^{\circ} \mathrm{C}$ (b) In situ scattering data of $2 \mathrm{NapVG}$ collected during the strain sweep showing no change in the scattering despite the gel being broken. (c) 2D scattering pattern of 2 NapVG at $0.1 \%$ strain and $1000 \%$ strain showing no alignment is caused by the application of shear during the experiment.

behaviour as there is little structure present (Figure 4b) and the data best fit to a power law (Table S7). Over time, cylindrical structures are formed and after 1 hour the data best fit to a flexible elliptical cylinder (Table S7). As for 2NapVG, there is an increase in scattering intensity with time (a slightly different $Q$ range was used for BrNapAV as the data were collected at a different beam line than the data for 
2NaVG and 2NapFF). The fits to the data imply only small changes to the radius and axis ratio, which occur as the network develops, meaning the fibres are not getting significantly thicker (Tables S7 and S8). Critically, the scattering does not change when the network synereses, showing that the structures forming the network are essentially unchanged when contraction occurs (Figure 4c). This shows that the syneresis is not due to a change in the morphology of the underlying structures.

After the gels have formed, it is possible to carry out standard frequency sweeps and strain sweeps whilst simultaneously measuring the SANS. This provides insight into how the gels respond to external stress. We exemplify this with data for a gel formed from 2NapVG after 12 hours. Data were collected under different applied strains (Figure 5 and Figures S10-S12). The applied strain was held for 1 hour to allow the scattering data to be collected. As expected for such gels, the gels break at a relatively low strain. Hence, the values of $G^{\prime}$ and $G$ " are essentially the same as the as-formed gel at low strains, but the gel breaks at $10 \%$ strain, with $G^{\prime \prime}$ becoming higher than $G$ 'at this value. The absolute values of $G$ ' and $G$ ' decrease at $100 \%$ and $1000 \%$ strain, with $G^{\prime}$ being higher than $G^{\prime}$ at these strains.

If the gel network breaks down into individual fibres, one would expect these to become aligned under the shear flow, ${ }^{29,33}$ and hence the SANS data should become anisotropic. This does not occur (Figure $5 \mathrm{c}$ ). However, fitting the SANS data collected at the different strains shows that at $0.1 \%$ strain and $1 \%$ strain, the parameters are essentially unchanged from the as-formed gel (note that the rheological data with time are collected at $0.1 \%$ strain), which makes sense for data collected in the linear viscoelastic region of the gel. At $10 \%$ strain, where the gel begins to break, the data can be fitted to a flexible elliptical cylinder model, but the radius increases from $2.8 \mathrm{~nm}$ (at $0.1 \%$ strain) to $3.2 \mathrm{~nm}$ (at $10 \%$ strain), with a similar axis ratio of 2.5 and 2.7, respectively. At higher strains, the radius increases further to 3.8 and $4.3 \mathrm{~nm}$ at $100 \%$ and $1000 \%$ strain, respectively, although the axis ratio remains at 2.5 and 2.8, respectively. Hence, as strain is applied, breaking the network, there is further lateral association of the fibrous structures.

Gel breakdown was examined by Yan et al. using rheoSANS. ${ }^{33}$ For their peptide-based gels, no anisotropy was observed under shear, showing that there is no alignment. The SANS data showed that there were no changes to the radius of the fibres as shear was applied. On the basis of these data, Yan et al. concluded that the network does not break down into individual fibres or small bundles under shear, but rather into domains of fibres. These domains mean that the gels are able to recover after shear as the domains immediately percolate when the shear is stopped.

An interesting observation here is that our data show different effects. Similar to the work of Yan et al., in that no alignment was observed, isotropic scattering data were observed at all strains. Hence, domains of fibres must be formed or else alignment would occur. However, the gels used here do not recover after flow, nor after application of a high strain. Hence, unlike the gels formed from MAX- 1 by Yan et al., it appears that in our systems, the applied strain drives an irreversible lateral association of the fibres and hence the gels cannot recover after cessation of the applied strain. This lateral association prevents the gel network from being able to reform after the applied strain is stopped. Our system remains as smaller bits of gel, rather than a bulk gel network. RheoSANS can therefore be used to explain why the gels used here do not recover unlike other examples.

\section{Conclusions}

We have shown here that we are able to monitor not only the change in structures that lead to gelation, but also how these structures directly link to the change in rheological properties as gelation occurs for different modified dipeptides. By using this in situ rheo-SANS method, not only have we been able to follow gelation of three different dipeptide gelators, but also access what is happening to the gels during syneresis and under increasing strain. We showed that syneresis is not due to any morphological change (for example, by a gel-to-gel transition) but rather is due to the contraction of the network with no changes to the scattering at the length-scales probed. Upon increasing strain, by rheology we can show that the gels break down, and by combining with SANS we can show that the gel breaks into domains of fibres. The fibres of the gels laterally associate and do not recover after this process. This gives us insight into why some gel systems do not recover after flow, which is vital when considering these materials for applications such as 3Dprinting and injection. As such, we have shown the power of using rheo-SANS to understand such low-molecular-weight gels. This method opens up ways to monitor gelation in other systems where the gelation happens over longer time periods, for example gels that are formed by a heat-cool cycle. For any system where there is a slow change in rheological properties, such as a change in viscosity, rheo-SANS could be used to assess how the structures have caused this change. One could also use this to assess aging and stability of systems where there is a change in rheological properties over time. We also highlight that this is not limited to SANS. Very recently, a rheosmall angle X-ray scattering (SAXS) study using GdL to slowly adjust the $\mathrm{pH}$ has been reported ${ }^{35}$ and so therefore there are many possibilities for this method for use in material analysis.

\section{Experimental Section}

All rheology data were collected on an Anton-Paar Physica MCR 501 rheometer with a customised titanium concentric cylinder geometry. Gelation was monitored over time by 
recorded $G^{\prime}$ and $G^{\prime \prime}$ at a strain of $0.5 \%$ and a frequency of $10 \mathrm{rad} / \mathrm{s}$ at a controlled temperature via a Peltier control and a water circulator. Strain data were collected at a set frequency of $10 \mathrm{rad} / \mathrm{s} . G^{\prime}$ and $G^{\prime \prime}$ data were collected at $0.1,1,10,100$, and $1000 \%$ strain for an hour each, whilst SANS data were simultaneously being collected.

In situ SANS data were then collected whilst the gelation was occurring in the rheometer. This was done at the ISIS Neutron and Muon source of the STFC Rutherford Appleton Laboratory, Didcot, United Kingdom (experiment number RB1910193), on the LOQ instrument (2NapVG and 2NapFF) and at the Institut Laue-Langevin Neutron Facility, Grenoble, France (experiment number 9-11-1801), on the D11 instrument. All SANS data were fitted using the SASView software.

$\mathrm{pH}$ data were collected using a HANNA instruments $\mathrm{pH}$ probe (FC200) with $6 \mathrm{~mm} \times 10 \mathrm{~mm}$ conical tip. Data were recorded every 30 seconds during gelation and logged using a custom-build data logger.

\section{Procedures}

2NapVG, 2NapFF, and BrNapAV were all synthesised according to previous literature procedures. ${ }^{20}$

2NapFF and 2NapVG gelator solutions were prepared at $10 \mathrm{mg} / \mathrm{mL}$, and BrNapAV at $5 \mathrm{mg} / \mathrm{mL}$. All were prepared by the addition of 1 molar equivalent of $\mathrm{NaOD}(0.1 \mathrm{M})$, then were then made up to the desired concentration with $\mathrm{D}_{2} \mathrm{O}$. The solutions were stirred overnight to ensure complete dissolution of the gelator. Next, the $\mathrm{pH}$ of each solution was adjusted, if required, to $\mathrm{pH} 11 \pm 0.1$.

To induce gelation, $8 \mathrm{mg} / \mathrm{mL}$ of GdL was added to the solutions. The solutions were then gently swirled until the GdL had dissolved. This could then be transferred into the rheometer for the rheo-SANS experiments or into a Sterlin vial for the $\mathrm{pH}$ measurements.

Full preparation and experimental details can be found in the Supporting Information.

\section{Funding Information}

Research Councils UK

Engineering and Physical Sciences Research Council

EP/L021978/1

$\mathrm{EP} / \mathrm{S} 032673 / 1$

Leverhulme Trust

ECF-2017-223

\section{Acknowledgments}

D.J.A. thanks the EPSRC for a fellowship (EP/L021978/1), which also funded K.M. L.T. thanks the University of
Glasgow for a PhD studentship. E.R.D. thanks the Leverhulme Trust for funding (ECF-2017-223), the University of Glasgow for an LKAS Leadership fellowship and the EPSRC for a New Investigator award (EP/S032673/1). The experiment at the Institut Laue-Langevin was allocated beam time under experiment number 9-11-1801 (DOI: 10.5291/ILLDATA.9-11-1801). The work at ISIS was allocated from the STFC, experiment number RB1910193. This work benefitted from SasView software, originally developed by the DANSE project under NSF award DMR-0520547. We thank Dr. Bart Dietrich for designing and building the custom-made data logger for the $\mathrm{pH}$ probe.

\section{Supporting Information}

Supporting Information for this article is available online at https://doi.org./10.1055/s-0040-1708832.

\section{Primary Data}

Primary data collected from scattering experiments can be found here DOI: 10.5291/ILL-DATA.9-11-1801.

\section{References}

(1) Fichman, G.; Gazit, E. Acta Biomater. 2014, 10, 1671.

(2) Du, X.; Zhou, J.; Shi, J.; Xu, B. Chem. Rev. 2015, 115, 13165.

(3) Draper, E. R.; Adams, D. J. Langmuir 2019, 35, 6506.

(4) Fleming, S.; Ulijn, R. V. Chem. Soc. Rev. 2014, 43, 8150.

(5) Martin, A. D.; Thordarson, P. J. Mater. Chem. B 2020, 8, 863.

(6) Orbach, R.; Adler-Abramovich, L.; Zigerson, S.; Mironi-Harpaz, I.; Seliktar, D.; Gazit, E. Biomacromolecules 2009, 10, 2646.

(7) Arakawa, H.; Takeda, K.; Higashi, S. L.; Shibata, A.; Kitamura, Y.; Ikeda, M. Polym. J. 2020, doi: 10.1038/s41428-019-0301-5.

(8) Yang, Z.; Liang, G.; Ma, M.; Gao, Y.; Xu, B.J.Mater.Chem. 2007, 17, 850.

(9) Ryan, D. M.; Nilsson, B. L. Polym. Chem. 2012, 3, 18.

(10) Kuang, Y.; Gao, Y.; Xu, B. Chem. Commun. 2011, 47, 12625.

(11) Mahler, A.; Reches, M.; Rechter, M.; Cohen, S.; Gazit, E. Adv. Mater. 2006, 18, 1365.

(12) Huang, R.; Qi, W.; Feng, L.; Su, R.; He, Z. Soft Matter 2011, 7, 6222.

(13) Nolan, M. C.; Fuentes Caparrós, A. M.; Dietrich, B.; Barrow, M.; Cross, E. R.; Bleuel, M.; King, S. M.; Adams, D. J. Soft Matter 2017, $13,8426$.

(14) Laverty, G.; McCloskey, A. P.; Gilmore, B. F.; Jones, D. S.; Zhou, J.; $\mathrm{Xu}, \mathrm{B}$. Biomacromolecules 2014, 15, 3429.

(15) Contreras-Montoya, R.; Castellví, A.; Escolano-Casado, G.; Juanhuix, J.; Conejero-Muriel, M.; Lopez-Lopez, M. T.; Cuerva, J. M.; Álvarez de Cienfuegos, L.; Gavira, J. A. Cryst Growth Des 2019, 19, 4229.

(16) Chakraborty, P.; Guterman, T.; Adadi, N.; Yadid, M.; Brosh, T.; Adler-Abramovich, L.; Dvir, T.; Gazit, E. ACS Nano 2019, 13, 163.

(17) Dudukovic, N. A.; Zukoski, C. F. Soft Matter 2015, 11, 7663.

(18) Jayawarna, V.; Ali, M.; Jowitt, T. A.; Miller, A. F.; Saiani, A.; Gough, J. E.; Ulijn, R. V. Adv. Mater. 2006, 18, 611. 
(19) Adams, D. J.; Butler, M. F.; Frith, W. J.; Kirkland, M.; Mullen, L.; Sanderson, P. Soft Matter 2009, 5, 1856.

(20) Draper, E. R.; Wallace, M.; Schweins, R.; Poole, R. J.; Adams, D. J. Langmuir 2017, 33, 2387.

(21) Wallace, M.; Iggo, J. A.; Adams, D. J. Soft Matter 2015, 11, 7739.

(22) Wang, Y.; Qi, W.; Wang, J.; Li, Q.; Yang, X.; Zhang, J.; Liu, X.; Huang, R.; Wang, M.; Su, R.; He, Z. Chem. Mater. 2018, 30, 7902.

(23) Tang, C.; Smith, A. M.; Collins, R. F.; Ulijn, R. V.; Saiani, A. Langmuir 2009, 25, 9447.

(24) Chen, L.; Revel, S.; Morris, K.; Serpell, L. C.; Adams, D. J. Langmuir 2010, 26, 13466.

(25) Draper, E. R.; Dietrich, B.; McAulay, K.; Brasnett, C.; Abdizadeh, H.; Patmanidis, I.; Marrink, S. J.; Su, H.; Cui, H.; Schweins, R.; Seddon, A.; Adams, D. J. Matter 2020, 2, 764.

(26) Cardoso, A. Z.; Mears, L. L. E.; Cattoz, B. N.; Griffiths, P. C.; Schweins, R.; Adams, D. J. Soft Matter 2016, 12, 3612.

(27) Draper, E. R.; Su, H.; Brasnett, C.; Poole, R. J.; Rogers, S.; Cui, H.; Seddon, A.; Adams, D. J. Angew. Chem. Int. Ed. 2017, 56, 10467.
(28) Martin, A. D.; Wojciechowski, J. P.; Robinson, A. B.; Heu, C.; Garvey, C. J.; Ratcliffe, J.; Waddington, L. J.; Gardiner, J.; Thordarson, P. Sci. Rep. 2017, 7, 43947.

(29) Eberle, A. P. R.; Porcar, L. Curr. Opin. Colloid Interface Sci. 2012, 17, 33.

(30) Blair, D. L. Chapter 2 Viscoelastic properties: the rheology of soft solids. In: Molecular Gels: Structure and Dynamics;. The Royal Society of Chemistry: London, 2018, 28-56.

(31) Riley, J. K.; Richards, J. J.; Wagner, N. J.; Butler, P. D. Soft Matter 2018, 14, 5344

(32) Dawn, A.; Kumari, H. Chem. Eur. J. 2018, 24, 762.

(33) Yan, C.; Altunbas, A.; Yucel, T.; Nagarkar, R. P.; Schneider, J. P.; Pochan, D. J. Soft Matter 2010, 6, 5143.

(34) Pocker, Y.; Green, E. J. Am. Chem. Soc. 1973, 95, 113.

(35) Ben Messaoud, G.; Le Griel, P.; Prévost, S.; Hermida-Merino, D.; Soetaert, W.; Roelants, S. L. V. K. Stevens, C. V.; Baccile, N. Soft Matter 2020, doi: 10.1039/C9SM02158B. 